\title{
Tat-PTD-Modified Oncolytic Adenovirus Driven by the SCG3 Promoter and ASH1 Enhancer for Neuroblastoma Therapy
}

\author{
Chuan Jin, ${ }^{*}$ Di Yu, ${ }^{*}$ Matko Čančer, Berith Nilsson, Justyna Leja, and Magnus Essand
}

\begin{abstract}
Secretogranin III (SGC3) belongs to the granin family and is highly expressed in endocrine and neural tissues. The human SCG3 promoter has not yet been characterized. We identified that a $0.5-\mathrm{kb}$ DNA fragment upstream of the SCG3 gene can selectively drive transgene expression in neuroblastoma cell lines. The strength of transgene expression was further increased, with specificity maintained, by addition of the human achaete-scute complex homolog 1 (ASH1) enhancer. We developed an oncolytic serotype 5-based adenovirus, in which the SCG3 promoter and ASH1 enhancer drive E1A gene expression. The virus was further modified with a cell-penetrating peptide (Tat-PTD) in the viral capsid, which we have previously shown results in increased adenovirus transduction efficiency of many neuroblastoma cell lines. The virus, Ad5PTD(ASH1-SCG3-E1A), shows selective and efficient killing of neuroblastoma cell lines in vitro, including cisplatin-, etoposide-, and doxorubicin-insensitive neuroblastoma cells. Furthermore, it delays tumor growth and thereby prolonged survival for nude mice harboring subcutaneous human neuroblastoma xenograft. In conclusion, we report a novel oncolytic adenovirus with potential use for neuroblastoma therapy.
\end{abstract}

\section{Introduction}

$\mathbf{N}$ EUROBLASTOMA Is an extracranial solid tumor, which originates from the sympathetic nervous system. The primary location is often along the path of neural crest-derived cells, typically in the adrenal medulla or paraspinal ganglia and as well as in the neck, chest, abdomen, or pelvis (Hoehner et al., 1996; Portela-Gomes et al., 2010). It is a disease of developing tissues, generally occurring in young children with a median age of 17 months at diagnosis (London et al., 2005). According to the new International Neuroblastoma Risk Group classification system (Monclair et al., 2009), neuroblastoma is divided into four broad categories. Some patients with neuroblastoma can regress spontaneously (Modak and Cheung, 2010). However, treatment of high-risk neuroblastoma still represents a significant challenge. Treatment includes resection of the primary tumor followed by high-dose chemotherapy (doseintensive cycles of cisplatin and etoposide alternating with vincristine, doxorubicin, and cyclophosphamide) and autologous bone marrow / hematopoietic stem cell rescue (Kushner et al., 1994; Maris, 2010). Most neuroblastoma cases initially respond well to chemotherapy, but ultimately $50-60 \%$ of patients with high-risk neuroblastoma relapse (Maris, 2010).
Oncolytic adenoviruses, in which the E1A gene and therefore viral replication is controlled by a tumor- or tissue-specific promoter, are emerging as therapeutic agents for cancer therapy (Essand, 2013). On replication, the virus lyses infected tumor cells and progeny virus generates a secondary infection of adjacent cells within the tumor. Infection with adenovirus serotype 5 (Ad5) is dependent on expression of the coxsackievirus-adenovirus receptor (CAR) on target cells, and many tumor cells downregulate CAR expression. To overcome this we have developed a capsid-modified Ad5 with the protein transduction domain (PTD) of the Tat protein from human immunodeficiency virus type 1 in the hexon hypervariable region 5 (HVR5). The transduction capacity and oncolytic efficacy of neuroblastoma cells were enhanced for Tat-PTD-modified Ad5 both in vitro and in vivo (Yu et al., 2011).

Secretogranin III (SCG3) is a member of the granin family and is involved in proteolytic processing in the regulated secretory pathway of neuroendocrine cells (Ozawa and Takata, 1995). Human SCG3 is expressed in neuroendocrine tumors, neuroblastoma (Portela-Gomes et al., 2010), as well as neuroendocrine cells of the heart, skeletal muscle, kidney, liver, and brain (Rong et al., 2002). The human SCG3 promoter has not yet been characterized. Achaete-scute-complex homolog 1 
(ASH1) is a member of the vertebrate proneural genes/ proteins expressed in neural crest cells and in early stages of CNS development, and plays an important role in differentiation of neurons (Chen et al., 1997). The ASH1 gene enhancer has been shown to provide cell type-specific expression in neuroblastoma cells (Watt et al., 2007).

We constructed an oncolytic Tat-PTD-based Ad5 in which E1A gene expression is driven by the SCG3 promoter and ASH1 enhancer. The virus shows selective and efficient killing of neuroblastoma cell lines, including cisplatininsensitive, doxorubicin-insensitive, and etoposide-insensitive neuroblastoma cells. Furthermore, it delays tumor growth and thereby prolonged survival for nude mice harboring human neuroblastoma.

\section{Materials and Methods}

\section{Cell lines and cytotoxic drugs}

Human neuroblastoma cell lines SH-SY-5Y, SK-N-Be(2), and SK-N-SH, and IMR-32 and SK-N-FI (a kind gift from F. Hedborg, Uppsala University, Uppsala, Sweden), were cultured in minimal essential medium (MEM) supplied with $10 \%$ fetal bovine serum (FBS), $1 \mathrm{mM}$ sodium pyruvate, penicillin $(100 \mathrm{IU} / \mathrm{ml})$, streptomycin $(100 \mu \mathrm{g} / \mathrm{ml})(1 \% \mathrm{PEST})$ and $1 \%$ nonessential amino acids. The neuroblastoma cell lines SK-N-DZ, SK-N-AS, and Kelly (gift from F. Hedborg) and the human lung non-small cell carcinoma line NCI-H727 (American Type Culture Collection [ATCC], Manassas, VA) were cultured in RPMI 1640 medium supplied with 10\% FBS, $1 \mathrm{mM}$ sodium pyruvate, and $1 \%$ PEST. The human pancreatic neuroendocrine tumor cell line BON (gift from J.C. Thompson and C.M. Townsend, Galveston, TX) was cultured in Dulbecco's modified Eagle's medium (DMEM) with GlutaMAX-I and F-12 nutrient mixture (Kaighn's modification) at a ratio of $1: 1$, supplemented with $10 \%$ FBS, $1 \mathrm{mM}$ sodium pyruvate, and 1\% PEST. The human lung carcinoma cell line A549 (ATCC), the human melanoma cell line Mel526 (gift from T. Boon, Ludwig Institute for Cancer Research [LICR], Brussels, Belgium), the human cervical cancer cell line $\mathrm{HeLa}$, the human foreskin fibroblast cell line 1064Sk (ATCC), and the adenovirus producer cell line 911 (Crucell, Leiden, The Netherlands) were cultured as previously described (Danielsson et al., 2008). All cell culture reagents were from Invitrogen (Carlsbad, CA) and all cell cultures were maintained in a $95 \%$ humidity incubator with a $5 \% \mathrm{CO}_{2}$ at- mosphere at $37^{\circ} \mathrm{C}$. Drug-insensitive neuroblastoma cell lines were established by repeated culture of cells with increasing concentrations of drugs for 3-5 days, followed by 1 week of recovery in drug-free medium when the cells were in an exponential growth phase (Iwasaki et al., 2002; Yan et al., 2007). A cisplatin-insensitive SK-N-FI clone was established, using up to $100 \mu M$ cisplatin (Meda, Solna, Sweden). Druginsensitive SH-SY-5Y clones were established by using up to $1 \mu M$ doxorubicin (Meda), $1 \mu M$ etoposide (Meda), and $0.2 \mu M$ vincristine (Hospira, Stockholm, Sweden).

\section{Recombinant adenoviruses}

All recombinant replication-deficient adenoviral vectors used in this study are based on human adenovirus serotype 5 (Ad5) and were constructed by AdEasy technology (He et al., 1998). The SCG3 promoter and ASH1 enhancer were obtained by PCR amplification from human genomic DNA (Roche, Mannheim, Germany), using primers described in Table 1. The PCR elements replaced the cytomegalovirus (CMV) promoter in pShuttle(CMV-Luc) (Cheng et al., 2004) to generate pShuttle(SCG3-Luc) and pShuttle(ASH1-SCG3-Luc), respectively. The Ad5 backbone vector pAdEasy(E3) used for homologous recombination to construct Ad5(SCG3-Luc) and Ad5(ASH1-SCG3-Luc) has been described previously (Leja et al., 2007; Danielsson et al., 2008), as has the construction of Ad5(MOCK) and Ad5(CMV-Luc) (Cheng et al., 2004).

A recombinant oncolytic adenovirus, Ad5PTD(ASH1SCG3-E1A), was constructed by recombineering (recombination-mediated genetic engineering) technology (Stanton et al., 2008). This virus contains both the E1B region and fulllength E3 region. Briefly, the als cassette, an expression cassette containing the ampicillin resistance gene $\left(A m p^{R}\right)$, the $l a c Z \alpha$ gene, and the sucrose-sensitive gene $s a c B$ gene, was amplified by PCR using primers pF.E1Ap-als and pR.E1Apals (Table 1). The PCR product replaced the E1A promoter in Ad5PTD(WT) (Yu et al., 2011) through a selection step based on Amp and LacZ as selection markers. The ASH1 enhancer-SCG3 promoter cassette was amplified with primers pF.E1Ap-SCG3 and pR.E1Ap-SCG3 (Table 1). The PCR product replaced the als cassette to generate Ad5PTD(ASH1-SCG3-E1A) through a counter-selection step based on sucrose-containing medium. Ad5PTD(GFP) (Yu et al., 2011), herein called Ad5PTD(MOCK), was used as a replication-deficient control virus for animal experiments.

Table 1. Primers Used in Study

\begin{tabular}{ll}
\hline Primer & \multicolumn{1}{c}{ Sequence $\left(5^{\prime} \rightarrow 3^{\prime}\right)$} \\
\hline pF-E4orf & CATCAGGTTGATTCACATCGG \\
pR-E4orf & AAGCGCTGTATGTTGTTCTG \\
pF-SCG3 & CTCGAGGATGCTTGATACGTCAACACA \\
pR-SCG3 & ACACTCGACGGTCAAGGGATAAG \\
pF-ASH1 & TTATAAATCTACAGGGTAATCTTGA \\
pR-ASH1 & CAGACAGCTTGCTCCATGTTAGGGAT \\
pF.E1Ap-als & TAATATGTCTCATTTCAGTCCCGGTGTCGGAGCGGCTCGGAGGAGAAAA \\
pR.E1Ap-als & CTCTACTCGCTGGCACTCAAGAGGGATCCCTGAGGTTCTTATGGCTCTTG \\
pF.E1Ap-SCG3 & GGCAGATAATATGTCTCATTTCAGTCCCGGTGTCGGAGCGGCTCGGAGGA \\
pR.E1Ap-SCG3 & GAAAACTCTACTCGCTGGCACTCAAGAGGCTTTTGTTATGAATGAAAAG \\
\hline & GGATCCCCTGTGACGGAAGATCACTTCG
\end{tabular}




\section{Virus production and titration}

All viral vectors and viruses used in this study are described in Fig. 1. Recombinant adenoviruses were produced in 911 cells and the viruses were purified by $\mathrm{CsCl}$ banding; dialyzed against a buffer containing $10 \mathrm{mM}$ Tris- $\mathrm{HCl}(\mathrm{pH}$ 8.0), $2 \mathrm{mM} \mathrm{MgCl}$, and $4 \%$ sucrose as previously described (Danielsson et al., 2008); and stored in aliquots at $-80^{\circ} \mathrm{C}$. Because the viral surface protein was modified in this study, the infectious viral titer depended on the cell line used for titration. Therefore, viral titers were determined by quantitative real-time PCR and defined as encapsidated viral genomes (EVG) per microliter (Yu et al., 2011).

\section{Luciferase assay}

Cells were transduced in suspension with Ad5(SCG3-Luc) and Ad5(ASH1-SCG3-Luc) at 100 EVG/cell in a total volume of $200 \mu \mathrm{l}$ for $2 \mathrm{hr}$ and cultured in 24-well plates. Total lysates were collected $48 \mathrm{hr}$ after transduction and luciferase assays were performed as described previously (Danielsson et al., 2008). Luciferase activity was calculated as relative light units (RLU) per milligram of total protein in the lysate and normalized to the luciferase activity from Ad5(CMV-Luc), set to $100 \%$.

\section{Cell viability assay}

Cells were transduced with viruses in suspension for $2 \mathrm{hr}$ with various EVG per cell and then seeded in 96-well plates in a total volume of $100 \mu$ l. Cells were treated with various concentrations of viruses or drugs. Cell viability was analyzed on day 4, using a CellTiter Aqueous one solution cell proliferation assay (MTS) kit (Promega, Madison, WI) according to the manufacturer's instructions. The relative cell viability was calculated on the basis of the ratio between the average absorbance for virus-transduced cells and the average for nontransduced cells. A time course experiment was performed for two neuroblastoma cell lines (SH-SY-5Y and SK-NFI) and one nonneuroblastoma cancer cell line (HeLa), using 10 EVG/cell. Cell viability was assayed on days 2, 4, and 6.

\section{Progeny virus assay as a measure of viral replication in various cell lines}

Cell lines were transduced with oncolytic adenoviruses and Ad5(MOCK) at 100 EVG/cell. The cells were washed with PBS twice after $2 \mathrm{hr}$ of transduction. Supernatants with progeny virus were collected after 4 days and added to 911 cells in 24-well plates for titration of functional infectious progeny virus. After $8 \mathrm{hr}$, the 911 cells were washed and harvested; viral DNA was isolated with a High Pure viral nucleic acid kit (Roche). The viral genome copy number in 911 cells was detected by quantitative real-time PCR as described previously (Yu et al., 2011). A time course experiment was performed for one neuroblastoma cell line (SK-N-FI), one nonneuroblastoma cancer cell line (HeLa), and normal fibroblasts (1064Sk), using $10 \mathrm{EVG} /$ cell. The production of progeny virus was assayed on days 2,4 , and 6 .

\section{Animal model for virus treatment}

Female, 3- to 4-week old, NMRI-nude mice were purchased from Taconic (Ry, Denmark). All mice were housed at

A

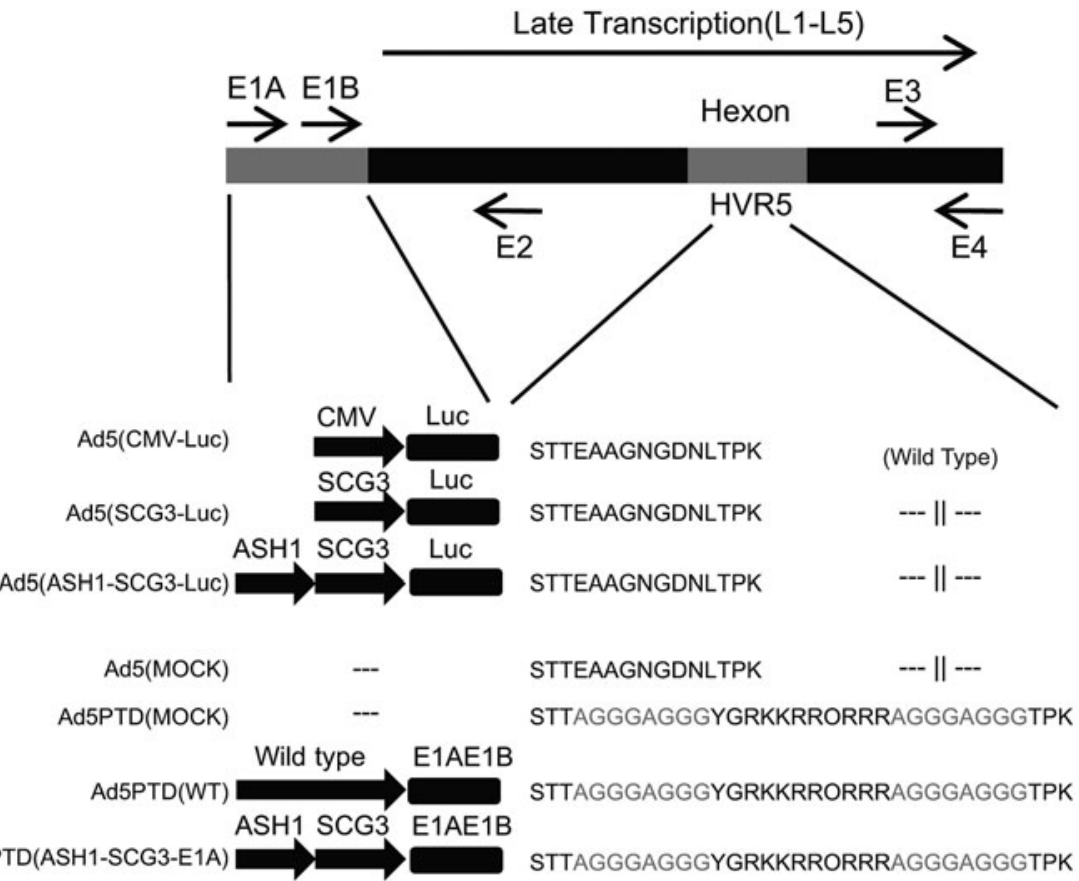

B ASH1 enhancer( 200bp)

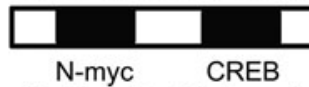

binding site binding site
SCG3 promoter( 500bp)

$\begin{array}{ccc}\text { GC box } & \text { TATA box } & \text { ATG } \\ \text { binding site } & \begin{array}{c}(-73 \text { to }-55 \mathrm{bp}) \\ \text { binding site }\end{array} & (0)\end{array}$

FIG. 1. Schematic illustration of vectors and viruses used in this study, with detailed illustrations of the SCG3 promoter and ASH1 enhancer. (A) Ad5(CMV-Luc), Ad5(SCG3-Luc), and Ad5(ASH1-SCG3-Luc) are E1-deleted Ad5-based vectors with luciferase gene expression controlled by the CMV promoter, the $0.5-\mathrm{kb}$ human SCG3 promoter, and the $0.5-\mathrm{kb}$ human SCG3 promoter and 0.2-kb human ASH1 enhancer, respectively. Ad5(MOCK) and Ad5PTD(MOCK) are E1-deleted replication-defective control viruses. The oncolytic adenoviruses Ad5PTD (WT) and AD5PTD(ASH1-SCG3-E1A) as well as Ad5PTD(MOCK) have a TatPTD modification in the hexon HVR5 region. (B) An illustration of the putative SCG3 promoter and ASH1 enhancer, which are used to control Luc or E1A expression. 
the Rudbeck Laboratory animal facility (Uppsala, Sweden) in individually ventilated cages (four mice per cage). SK-N-FI cells were mixed at a 1:1 volume ratio with Matrigel (BD Biosciences, San Jose, CA) and $1 \times 10^{6}$ cells were injected subcutaneously, in a total volume of $100 \mu \mathrm{l}$, into the right hind flank. Mice were treated with peritumoral injections of either Ad5PTD(MOCK) as control or Ad5PTD(ASH1-SCG3E1A) on days 7,13 , and 18 at a dose of $5 \times 10^{9}$ EVG/injection in $30 \mu$ l. Six mice per group were used. Tumor growth was monitored by caliper measurement. Tumor volume was calculated according to the ellipsoid volume formula (length $\times$ width $\times$ depth $\times \pi / 6$ ). Mice were killed if the tumor volume grew to greater than $900 \mathrm{~mm}^{3}$. The experiment was terminated after the last mouse was killed in the Ad5PTD(MOCK) group.

In another experimental setting, tumor-bearing NMRInude mice (three per group) were treated with either Ad5PTD(MOCK) or Ad5PTD(ASH1-SCG3-E1A) as described previously and killed 3 days after the last virus injection (on day 21). Tumor and liver samples from each mouse were harvested, frozen, or fixed in $4 \%$ formalin. Viral genome copy number was detected by quantitative real-time PCR from $10 \mathrm{mg}$ of frozen tumor and liver sample as described previously (Yu et al., 2013). Formalin-fixed tumor samples were embedded in paraffin and sectioned (thickness, $5 \mu \mathrm{m}$ ) at various tumor depths. For immunofluorescence analysis of viral protein, sections were immunofluorescently stained with primary mouse anti-hexon antibody (cat. no. MAB8052; Millipore, Billerica, MA) and a secondary fluorescein isothiocyanate (FITC)-conjugated rabbit $\mathrm{F}\left(\mathrm{ab}^{\prime}\right)_{2}$ fragment anti-mouse immunoglobulin (cat. no. F0313; Dako, Glostrup, Denmark) to detect viral hexon protein. They were also stained with Hoechst 33342 (Invitrogen) for nuclei. Images were captured with a Zeiss Axio Imager M2 (Carl Zeiss, Jena, Germany). For evaluation of necrosis, sections were stained with hematoxylin-eosin before an experienced pathologist blindly graded the tumor sections for percentage of necrosis (10\% intervals; at least 10 slides for each group).

\section{Statistical analysis}

Statistical analysis was performed with GraphPad Prism software version 5.01 (GraphPad Software, San Diego, CA). An unpaired Student $t$ test was used for comparison in the luciferase assay, MTS cell-killing assay, progeny virus assay, viral genome copies in tumor and liver, and the percentage of necrosis area in tumors. The log-rank test was used to compare survival curves created by the Kaplan-Meier method.

\section{Biosafety level and ethics declaration}

The Swedish Work Environment Authority has approved the work with genetic modification of the infectious capacity of human adenovirus serotype 5 (ID number 202100-2932 v66a13 [laboratory] and v67a9 [mice]) and genetic modification of replication capacity of human adenovirus serotype 5 (ID number 202100-2932 v66a11 [laboratory] and v67a7 [mice]). All experiments regarding modified adenovirus were conducted under biosafety level 2. The Uppsala Animal Ethics Committee has approved the animal studies (ID number C319/9).

\section{Results}

Oncolytic adenovirus with SCG3 promoter and ASH1 enhancer selectively kills neuroblastoma cells

To develop a neuroblastoma-specific adenovirus we cloned 2-, 1-, and 0.5-kb genomic $5^{\prime}$ flanking sequences of the SCG3 and NESP55 genes as well as the 0.9-kb SCG2 promoter (Li et al., 2008) into green fluorescent protein (GFP) expression plasmids and transfected cell lines of various origin (Supplementary Fig. S1A; supplementary data are available online at www.liebertonline.com/hum). Taking into consideration the activity of the promoter elements together with the sizes of the elements (space limitations in the adenoviral genome), we decided to continue with the $0.5-\mathrm{kb}$ SCG3 and 0.5-kb NESP55 promoters and introduced the 0.2$\mathrm{kb}$ ASH1 enhancer for further screening (Supplementary Fig. $\mathrm{S} 1 \mathrm{~B})$. On the basis of the results we then decided to continue with the $0.5-\mathrm{kb}$ SCG3 promoter and the 0.2-kb ASH1 enhancer element. Although the human SCG3 promoter has not been characterized, we found that there is a putative TATA box-binding site and a putative GC box-binding site within the $0.5-\mathrm{kb}$ region upstream of the SCG3 gene (Fig. 1B). The human ASH1 enhancer contains a putative N-Mycbinding site and a putative CREB-binding site (Fig. 1B).

We constructed two replication-deficient Ad5 vectors in which luciferase (Luc) gene expression is controlled by either the $0.5-\mathrm{kb}$ SCG3 promoter, Ad5(SCG3-Luc), or the $0.5-\mathrm{kb}$ SCG3 promoter and the 0.2-kb ASH1 enhancer, Ad5(ASH1SCG3-Luc). We found that the SCG3 promoter had the highest activity in the following neuroblastoma cell lines: SKN-Be(2), IMR-32, SK-N-DZ, SK-N-AS, and SK-N-FI, whereas it had rather low activity in the Kelly cell line and very low activity in SH-SY-5Y cells (Supplementary Fig. S2). However, the luciferase activity obtained for the recombinant ASH1SCG3 promoter, expressed as a percentage of CMV promoter activity, is in line with previous findings for human tissuespecific promoters (Danielsson et al., 2008). We found low if any expression in all the nonneuroblastoma tumor cell lines: HeLa (cervical cancer), lung carcinoma (A549), and Mel526 (melanoma) and in normal cells (1064Sk; foreskin fibroblasts) (Supplementary Fig. S2). Furthermore, the ASH1 enhancer significantly improved SCG3 promoter activity in the neuroblastoma cell lines Kelly $(p=0.006)$ and SK-N-FI $(p=0.018)$ (Supplementary Fig. S2).

To investigate the killing ability of an oncolytic virus controlled by the SCG3 promoter and ASH1 enhancer, Ad5PTD(ASH1-SCG3-E1A), in which the ASH1-SCG3 sequence controls E1A expression, was constructed. This virus contains the full-length E1B region and the viral capsid is modified with PTD sequences in the hexon HVR5. Ad5PTD(ASH1-SCG3-E1A) was evaluated side-by-side with Ad5(MOCK) and Ad5PTD(WT). Cell lines were transduced at various EVG/cell concentrations and relative cell viability was measured after 4 days (Fig. 2A). Ad5PTD(ASH1-SCG3E1A) killed all evaluated neuroblastoma cell lines: SH-SY-5Y, IMR-32, SK-N-DZ, SK-N-AS, Kelly, SK-N-FI, and SK-N$\mathrm{SH}$ significantly better than did Ad5(MOCK), whereas it had nearly no lytic activity in the nonneuroblastoma cell lines 1064Sk, A549, Mel526, and HeLa, not significantly different from Ad5(MOCK). Ad5PTD(WT), which was used as a positive control, showed killing ability in all cell lines without any selectivity. These results suggest that 


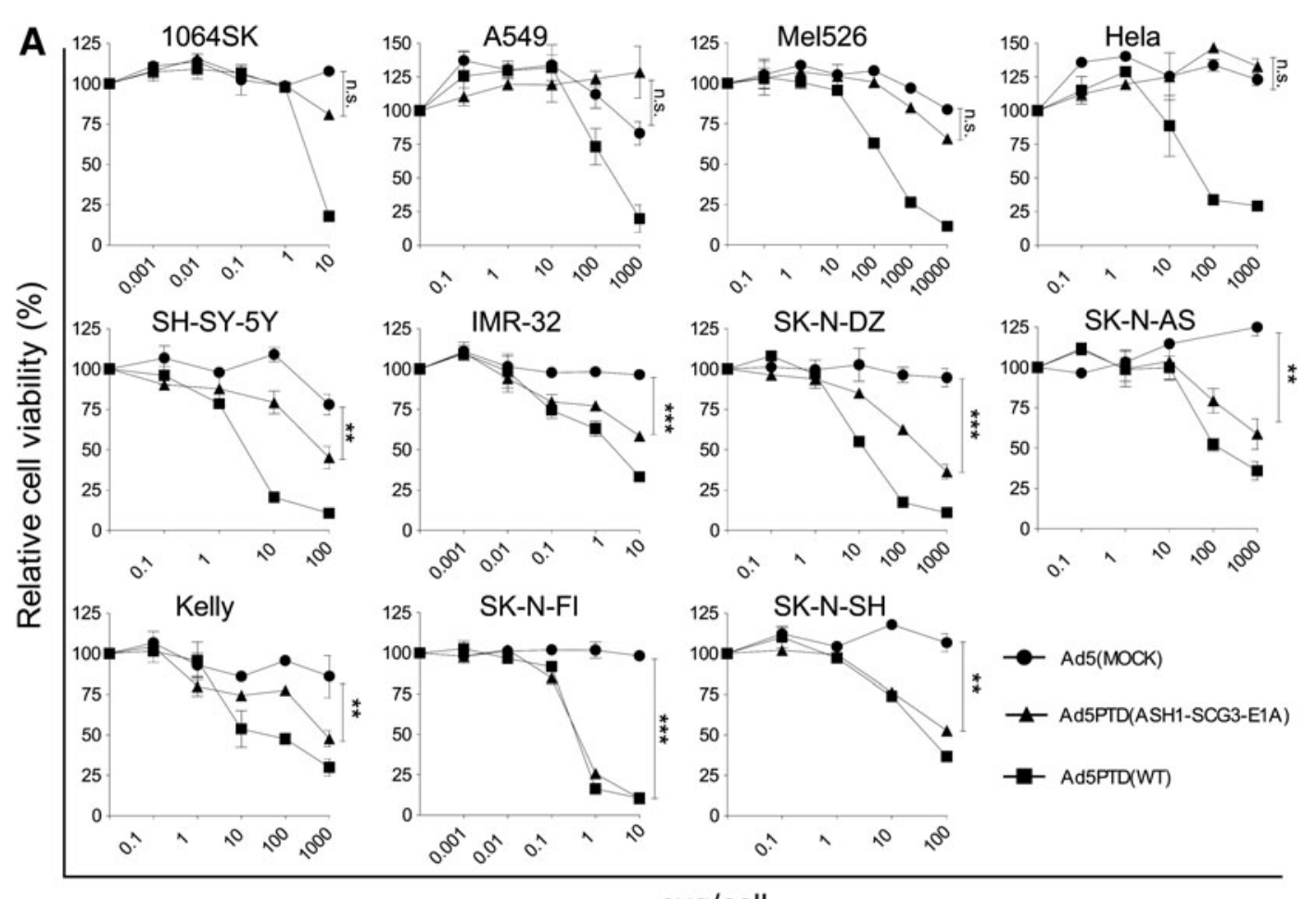

evg/cell

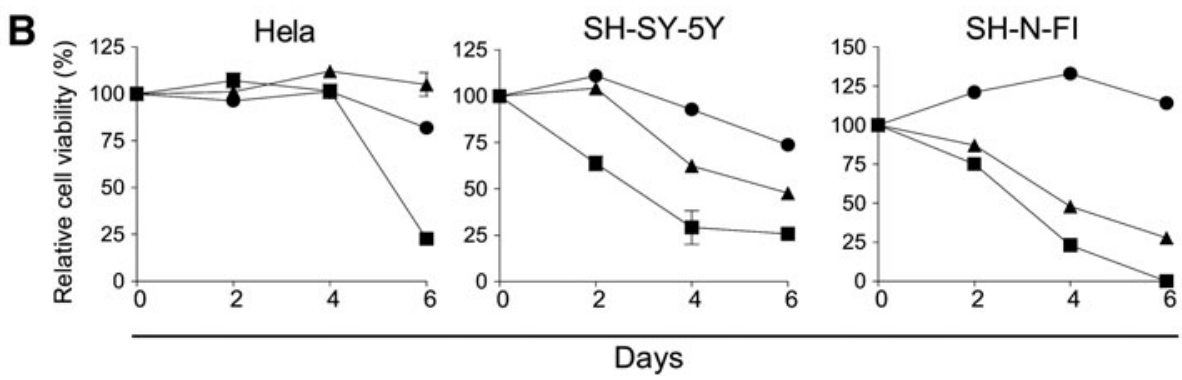

FIG. 2. Ad5PTD(ASH1-SCG3-E1A) possesses selective killing of neuroblastoma cells. (A) Neuroblastoma cell lines (SH-SY5Y, IMR-32, SK-N-DZ, SK-N-AS, Kelly, SK-N-FI, SK-N-SH), nonneuroblastoma tumor cell lines (Mel526, A549, HeLa), and normal fibroblast cells (1064Sk) were transduced with Ad5PTD(ASH1-SCG3-E1), Ad5PTD(WT), or Ad5(MOCK) virus at various encapsidated viral genome (EVG)/cell. Relative cell viability was analyzed by MTS assay 4 days after virus transduction. (B) Three cell lines: SK-N-FI and SH-SY-5Y (neuroblastoma), and HeLa (nonneuroblastoma tumor) were transduced with Ad5PTD(ASH1-SCG3-E1), Ad5PTD(WT), or Ad5(MOCK) at 10 EVG/cell and relative cell viability was analyzed by MTS assay on days 2, 4, and 6 . All data are shown as means \pm SD from three independent experiments, each with triplicate samples $\left({ }^{* *} p<0.01,{ }^{* * *} p<0.001 ;\right.$ n.s., not significant).

Ad5PTD(ASH1-SCG3-E1A) is capable of efficient and selective killing of neuroblastoma cells. Three cell lines: SK-N-FI and SH-SY-5Y (neuroblastoma), and HeLa (nonneuroblastoma tumor), were also transduced at $10 \mathrm{EVG} /$ cell and analyzed at various time points with specificity retained (Fig. 2B).

\section{Ad5PTD(ASH1-SCG3-E1A) is capable of specific replication in neuroblastoma cell lines}

The replication ability of Ad5(MOCK), Ad5PTD(ASH1SCG3-E1A), and Ad5PTD(WT) was evaluated in the same panel of cell lines. All cell lines were infected with virus at 100 EVG/cell, and supernatant containing progeny virus was collected after 4 days and added to 911 cells to detect viral genomes from fully functional progeny viral particles. Ad5PTD(ASH1-SCG3-E1A) replicated well in most neuroblas- toma cell lines, in which the DNA of functional virus was significant higher than for Ad5(MOCK) in all cell lines (Fig. 3A). In three of the five evaluated neuroblastoma cell lines Ad5PTD(ASH1-SCG3-E1A) replicated as efficiently as Ad5PTD (WT), whereas in two of the cell lines the yield of Ad5PTD(ASH1-SCG3-E1A) progeny virus was somewhat lower than for Ad5PTD(WT), probably because of weaker promoter activity (ASH1-SCG3 vs. wild-type E1A). Importantly, replication of Ad5PTD(ASH1-SCG3-E1A) in the nonneuroblastoma cell lines (1064Sk, A549, Mel526, HeLa) was not significantly different from Ad5(MOCK). Ad5PTD(WT) showed efficient but nonspecific replication ability in all evaluated cell lines. Three cell lines: SK-N-FI (neuroblastoma), HeLa (nonneuroblastoma tumor), and 1064Sk (normal fibroblasts), were also transduced at $10 \mathrm{EVG} /$ cell and progeny virus was harvested and titrated at various time points (Fig. 3B). Taken together, 


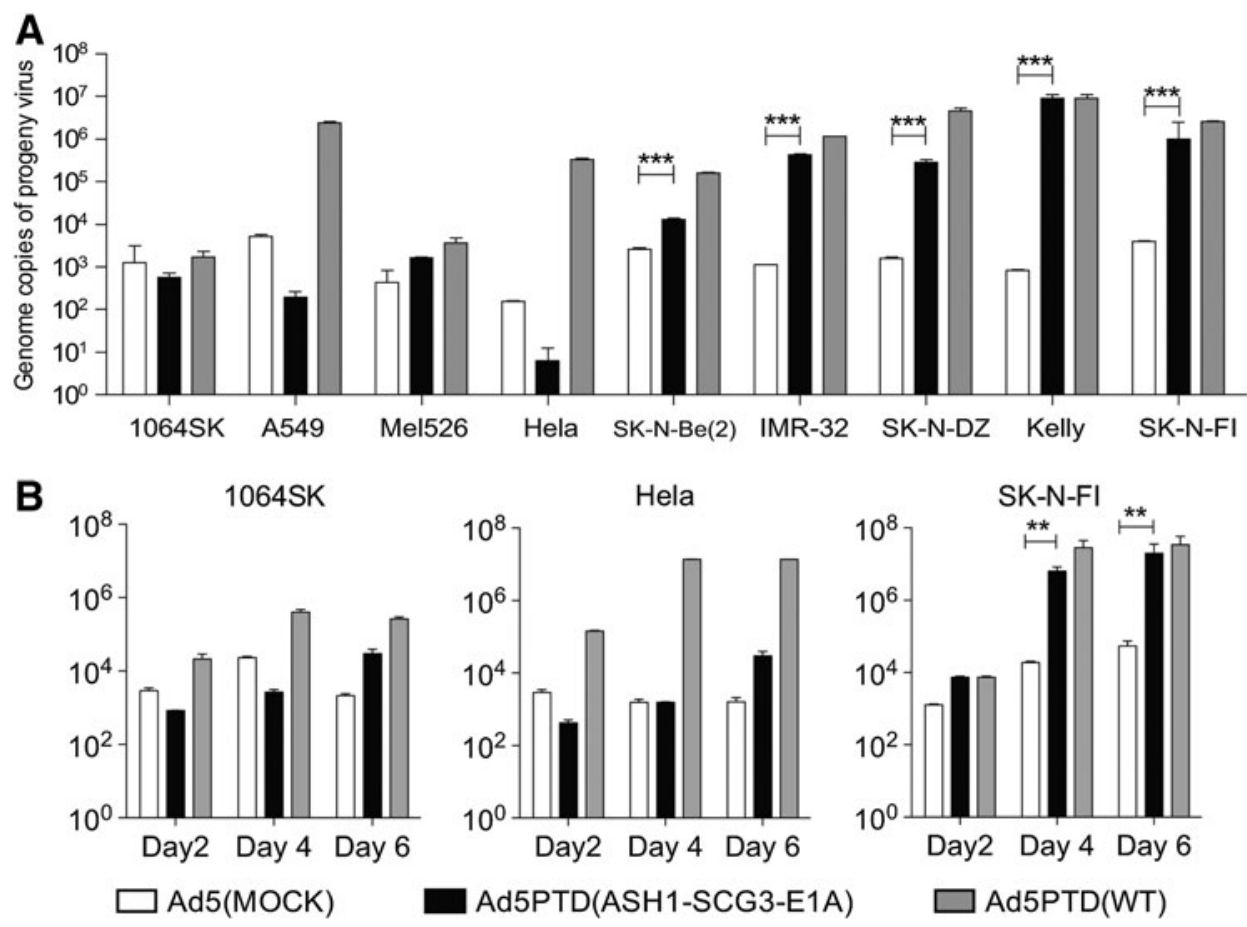

FIG. 3. Ad5PTD(ASH1-SCG3-E1A) shows selective replication in neuroblastoma cell lines. (A) Neuroblastoma cell lines and a nonneuroblastoma cell line were transduced with Ad5PTD(ASH1-SCG3-E1), Ad5PTD(WT), or Ad5(MOCK) at 100 EVG/ cell. Supernatants with progeny virus were collected after 4 days and added to 911 cells for titration of infectious progeny virus. (B) Three cell lines: SK-N-FI (neuroblastoma), HeLa (nonneuroblastoma tumor), and 1064Sk (normal fibroblasts) were transduced with Ad5PTD(ASH1-SCG3-E1A), Ad5PTD(WT), or Ad5(MOCK) at 10 EVG/cell; supernatants with progeny virus were harvested at various time points and added to 911 cells for titration of infectious progeny virus. (A and B) 911 cells exposed to progeny virus were harvested after $8 \mathrm{hr}$, followed by viral DNA isolation. Viral copies were determined by quantitative real-time PCR analysis. All data are shown as means + SD from three independent experiments, each with triplicate samples, $\left({ }^{* *} p<0.01,{ }^{* * *} p<0.001\right)$.

these data further strengthen the notion that Ad5PTD(ASH1SCG3-E1A) is both effective and selective for neuroblastoma cells.

\section{Oncolytic Ad5PTD(ASH1-SCG3-E1A) virus kills chemotherapeutic drug-insensitive neuroblastoma cells}

A cisplatin-insensitive clone of the SK-N-FI neuroblastoma cell line and doxorubicin-, etoposide-, and vincristineinsensitive clones of SH-SY-5Y were established and treated with viruses at various EVG/cell concentrations or with drugs at various concentrations. Relative cell viability was analyzed by MTS assay. Ad5PTD(ASH1-SCG3-E1A) killed cisplatin-sensitive and insensitive SK-N-FI cells with the same efficacy ( $\mathrm{IC}_{50}$ of $6 \mathrm{EVG} /$ cell), whereas 12-fold more cisplatin was needed to kill cisplatin-insensitive SK-N-FI cells $\left(\mathrm{IC}_{50}\right.$ of $\left.100 \mu \mathrm{M}\right)$ compared with cisplatin-sensitive SK-N-FI cells (IC 50 of $8 \mu \mathrm{M}$ ) (Fig. 4A). Ad5PTD(WT) also killed cisplatin-sensitive and insensitive SK-N-FI with the same efficacy (Fig. 4A). The adenovirus killing efficiency of etoposide-insensitive SH-SY-5Y (Fig. 4B) and doxorubicininsensitive SH-SY-5Y (Fig. 4C) was reduced to some extent compared with the corresponding drug-insensitive cells. In contrast, vincristine-insensitive SH-SY-5Y cells proved difficult to kill both with $\operatorname{Ad5PTD(ASH1-SCG3-E1A)~and~}$ Ad5PTD(WT) (Fig. 4D).

\section{Ad5PTD(ASH1-SCG3-E1A) suppresses SK-N-FI neuroblastoma tumor growth in nude mice and prolongs survival}

We next wanted to examine the efficacy of Ad5PTD(ASH1SCG3-E1A) to control tumor growth in vivo. SK-N-FI cells were injected subcutaneously into nude mice and established tumors were treated on day 7 by peritumoral injection of either Ad5PTD(MOCK) or Ad5PTD(ASH1-SCG3-E1A). The tumor-bearing mice were treated again on days 13 and 18 . Mice treated with Ad5PTD(ASH1-SCG3-E1A) exhibited suppression of tumor growth compared with mice treated with Ad5PTD(MOCK). The last mouse in the Ad5PTD(MOCK)treated group had to be killed on day 54, whereas four of six mice from the Ad5PTD(ASH1-SCG3-E1A)-treated group were alive at 60 days, when the experiment was terminated. All mice had tumors at the time of termination, but only one of the four mice had a growing tumor. Compared as two groups of mice, Ad5PTD(ASH1-SCG3-E1A)-treated mice had significantly smaller tumors than did Ad5PTD(MOCK)-treated mice (Fig. 5A). Furthermore, mice treated with Ad5PTD(ASH1SCG3-E1A) showed significantly prolonged survival compared with Ad5PTD(MOCK)-treated mice (Fig. 5B).

In another experiment, mice were treated with either Ad5PTD(MOCK) or Ad5PTD(ASH1-SCG3-E1A) in the same manner and killed 3 days after the last treatment for analysis. A significantly higher copy number of viral genome 
A
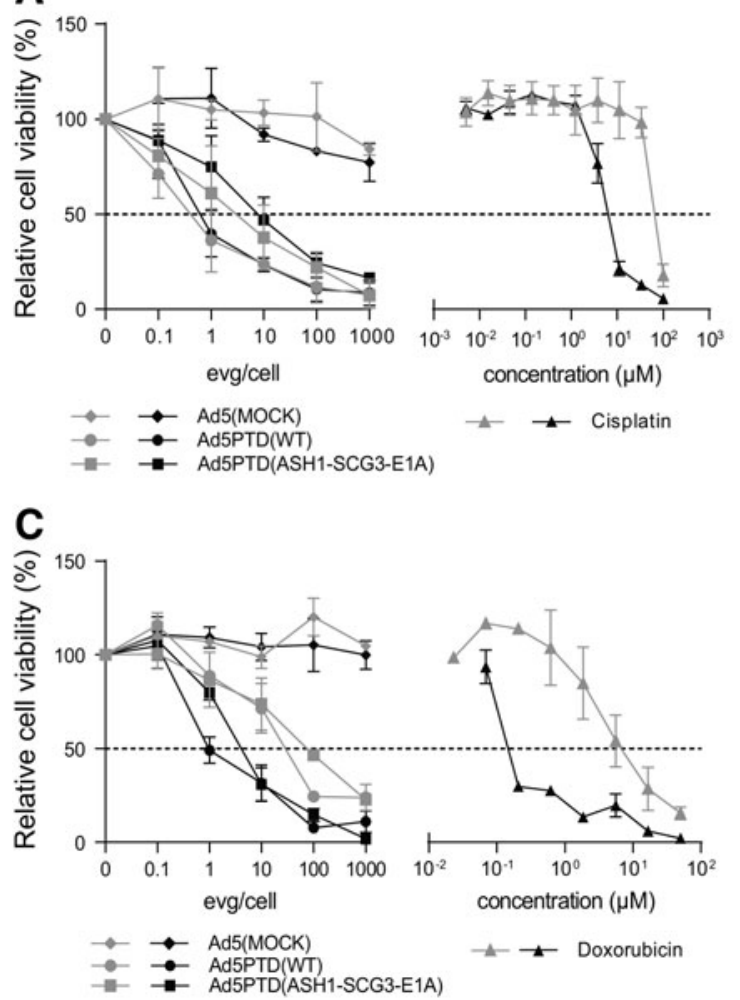

B
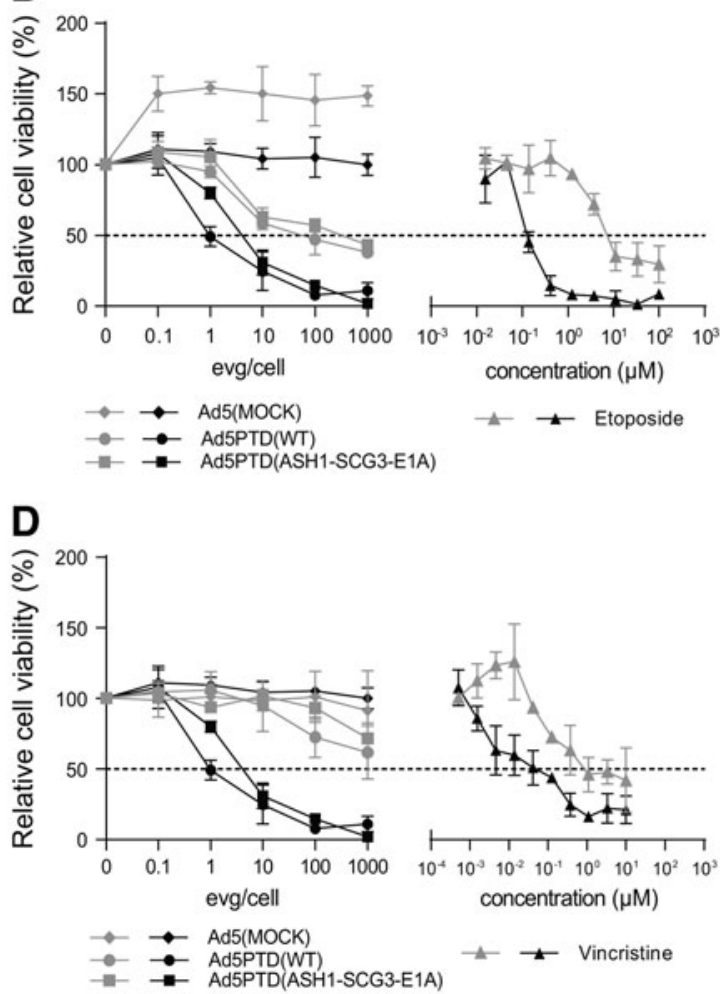

FIG. 4. Ad5PTD(ASH1-SCG3-E1A) kills drug-insensitive neuroblastoma cells expressing neural stem cell markers. A cisplatin-insensitive clone of SK-N-FI and doxorubicin-insensitive, etoposide-insensitive, and vincristine-insensitive clones of SH-SY-5Y were established through repeated cycles of culture with increasing drug concentration. (A-D) Drug-sensitive (black lines) and drug-insensitive (gray lines) neuroblastoma cells were treated with virus or drugs. Relative cell viability was analyzed after 4 days by MTS assay. (A) Oncolytic virus and cisplatin killing of SK-N-FI cells. (B) Oncolytic virus and etoposide killing of SH-SY-5Y cells. (C) Oncolytic virus and doxorubicin killing of SH-SY-5Y cells. (D) Oncolytic virus and vincristine killing of SH-SY-5Y cells. All data are shown as means \pm SD from four independent experiments, each with triplicate samples.

DNA was detected in tumors treated with Ad5PTD(ASH1SCG3-E1A) than in tumors treated with Ad5PTD(MOCK) (Fig. 5C), indicating Ad5PTD(ASH1-SCG3-E1A) replication in tumors. However, the copy number of viral genome DNA in liver was similar for both treatment groups (Fig. 5D), indicating that there is little leakage of Ad5PTD(ASH1SCG3-E1A) into the circulation even though the virus replicates in tumor. Hexon protein staining localized within the nuclei of cells (Fig. 5E), depicting the assembly of progeny viral particles (Wodrich et al., 2003), which further indicates replication of Ad5PTD(ASH1-SCG3-E1A) in vivo. As expected, hexon protein could not be detected in tumors treated with the nonreplicative $\operatorname{Ad5PTD(MOCK)~virus~al-~}$ though large areas were analyzed (Fig. 5E). Furthermore, tumors treated with Ad5PTD(ASH1-SCG3-E1A) had a significantly higher percentage of necrotic areas compared with Ad5PTD(MOCK)-treated tumors (Fig. 5F). Representative high-magnification images of treated tumors are shown in Fig. 5G. In the Ad5PTD(MOCK)-treated tumor group, xenotransplanted tumors were well structured with few necrotic areas $(<10 \%)$, with most of the tumor cells alive and cells undergoing mitosis as indicated by arrows (Fig. 5G, top). In contrast, tumors treated with Ad5PTD(ASH1SCG3-E1A) presented high amounts of necrosis (about 30\%) and disruption of the cellular structures (Fig. 5G, bottom).
Infiltrating blood cells were also observed within necrotic areas.

\section{Discussion}

Oncolytic adenoviruses have already been clinically used at two centers for the treatment of children with metastatic neuroblastoma (Garcia-Castro et al., 2010; Pesonen et al., 2010). In one study, a 6-year-old boy with lymph node and bone marrow metastases was treated by injection of Ad5/3Cox2L $\Delta 24$ oncolytic adenovirus, which is an Ad5 virus with Ad3 fiber knob and a 24-bp deleted $(\Delta 24)$ E1A gene driven by the cyclooxygenase-2 (COX2) promoter. The treatment was found to be safe and at 3 months, a bone marrow biopsy indicated a minimal number of tumor cells in a mostly healthy marrow (Pesonen et al., 2010). In the other study, four children with metastatic neuroblastoma refractory to first-line therapies received several doses of autologous mesenchymal stem cells loaded with oncolytic adenovirus (Garcia-Castro et al., 2010). The adenovirus used in this case was an Ad5 virus with an RGD peptide in the fiber knob, which binds to cellular integrin, and it had the 24-bp deleted $(\Delta 24)$ E1A gene driven by the E2F-1 promoter. Tolerance to the treatment was excellent. A complete clinical response was documented in one case, and the child is in 

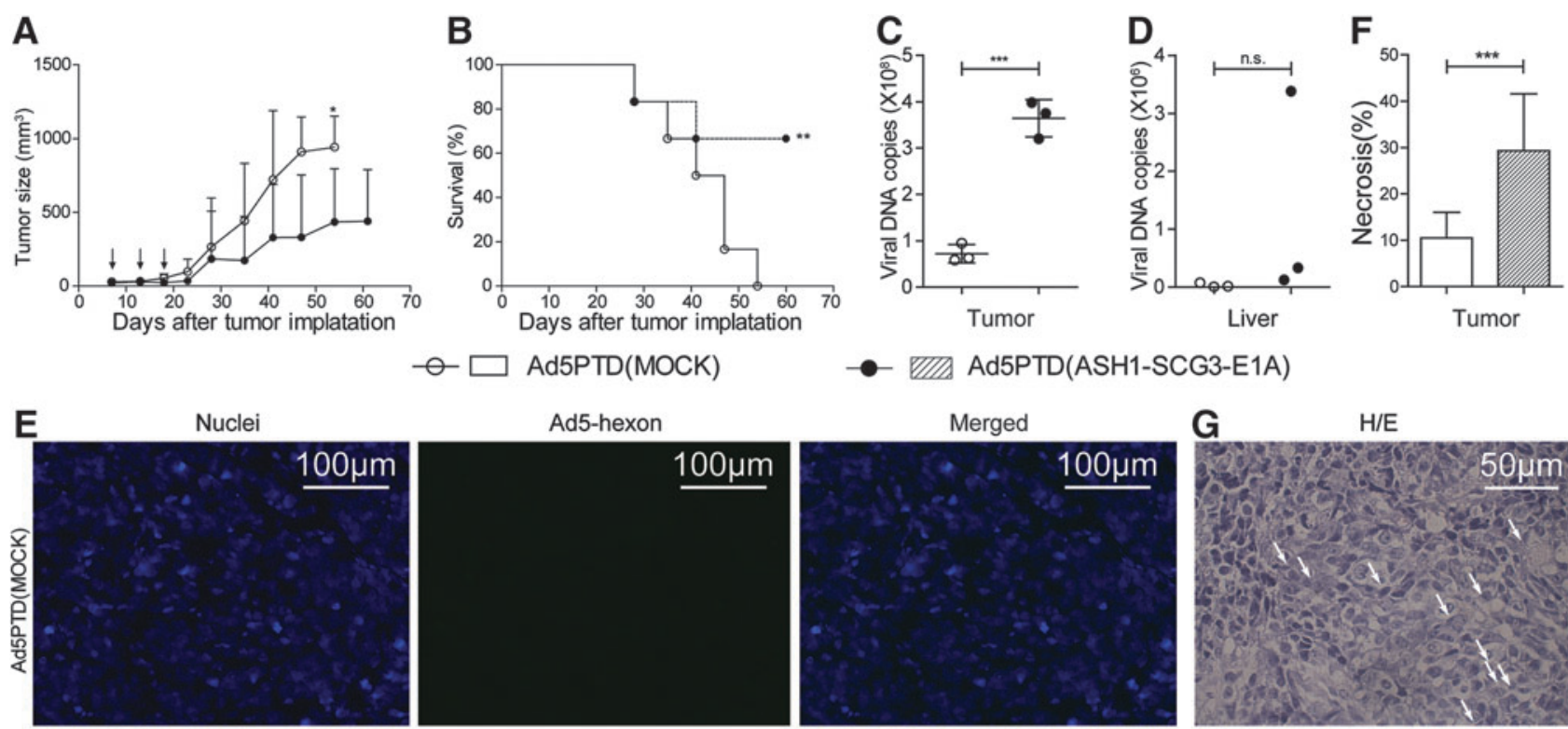

$\multimap \square \operatorname{Ad5PTD(MOCK)}$

IIA Ad5PTD(ASH1-SCG3-E1A)
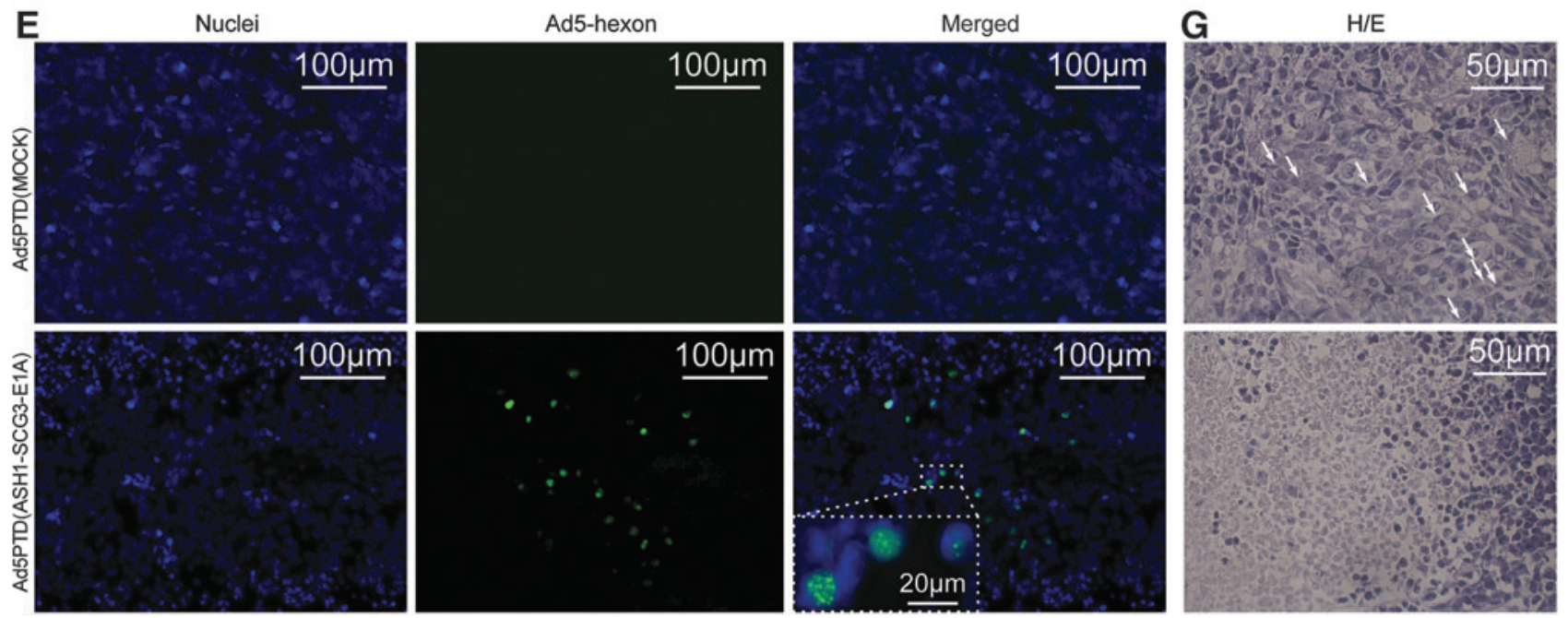

FIG. 5. Treatment with Ad5PTD(ASH1-SCG3-E1A) delays tumor growth and prolongs survival of nude mice with transplanted human neuroblastoma tumor. NMRI-nude mice harboring subcutaneous human neuroblastoma (SK-N-FI) tumors were treated by peritumoral virus injection on days 7,13, and 18 after tumor implantation. Tumors were monitored by caliper measurements until the tumor size reached $900 \mathrm{~mm}^{3}$, at which point the mice were killed. The experiment was terminated on day 60 after tumor implantation. (A) Tumor sizes for the two groups of treated mice are shown as means $+\mathrm{SD}\left(n=6\right.$, $\left.{ }^{*} p<0.05\right)$. (B) A Kaplan-Meier survival curve shows survival data. The log-rank test was used to compare survival curves ( $n=6$, ${ }^{* *} p<0.01$ ). Three mice from each treatment group were killed 3 days after the last virus injection. Tumor (C) and liver (D) samples were subjected to quantitative PCR for viral DNA detection. Data are shown as DNA copy number per milligram of tissue harvested from each mouse $(* * *<0.001$; n.s., $p>0.05)$. (E) Tumor samples stained to visualize (original magnification, $\times 200$ ) viral hexon protein (FITC, green) and nuclei (Hoechst 33342, blue). Colocalization of hexon and nuclei in Ad5PTD(ASH1-SCG3-E1A)-treated tumor was visualized at an original magnification of $\times 630$. (F) Tumor samples were stained with hematoxylin and eosin $(\mathrm{H} / \mathrm{E})$ to visualize necrosis. The percentages of tumor necrosis were blindly graded with $10 \%$ intervals from at least three sections per tumor sample at various sites in the tumors. Data are shown as the mean percentage necrotic area with error bars indicating the SD $(* * * p<0.001)$. (G) Representative pictures of H/E staining of tumors from either treatment group as indicated. Arrows show mitotic cells.

complete remission 3 years after this therapy. Taken together, these two studies suggest that oncolytic viruses can have substantial effects on otherwise treatment-resistant neuroblastoma.

We have previously developed a neuroendocrine-specific oncolytic Ad5 virus driven by the chromogranin A (CgA) promoter and shown that it is able to efficiently kill neuroendocrine tumor cells both in vitro and in vivo (Leja et al., 2007). The CgA promoter works well in neuroendocrine tumors and to some extent in neuroblastoma (Leja et al., 2007, 2010). In this study we wanted to investigate other closely related promoters with the aim to develop a neuroblastomaspecific oncolytic adenovirus. We investigated the NESP-55, secretogranin II (SCG2), and secretogranin III (SCG3) promoters for their selective expression in neuroblastoma cells. All promoters had good specificity and activity in neuroblastoma cells, but among them we decided to continue with the $0.5-\mathrm{kb}$
SCG3 promoter and to include the 0.2-kb ASH1 enhancer. Secretogranin III expression has been reported in neuroendocrine tumors and neuroblastoma (Portela-Gomes et al., 2010). Human ASH1 shows tissue-specific expression in neuroendocrine tumors (Chen et al., 1997). Many primary neuroblastoma cells and cell lines possess an immature phenotype, and express high levels of human ASH1 (Gestblom et al., 1999). The choices of regulatory elements therefore seemed rational to obtain neuroblastoma specificity. We also found that the ASH1 element enhances SCG3 promoter activity in neuroblastoma cell lines, significantly so in Kelly and SK-N-FI cells.

We have previously shown that Tat-PTD modification of the Ad5 hexon dramatically increases the transduction capacity of CAR-negative/low cell lines and that Tat-PTDmodified oncolytic viruses have therapeutic effects in animal models of human neuroblastoma and neuroendocrine tumors (Yu et al., 2011). However, although our previously 
reported Tat-PTD-modified oncolytic virus has high transduction efficiency and dramatic therapeutic effects, it does not have tissue selectivity ( $\mathrm{Yu}$ et al., 2011). In the present study, we therefore combined a tissue-specific promoter with the Tat-PTD modification to produce a highly infectious oncolytic adenovirus with selective activity in neuroblastoma. Our animal studies clearly show that the Ad5PTD(ASH1-SCG3-E1A) virus has oncolytic activity in vivo when it is injected into established SK-N-FI tumors. SK-N-FI xenografts are fast-growing and challenging. When treated with Ad5PTD(MOCK) the mice must be killed within 50 days. The majority of mice treated with Ad5PTD(ASH1SCG3-E1A) were still alive after 60 days and the growth of tumor was significantly delayed. We observed wellstructured tumors in the $\operatorname{Ad5PTD}(\mathrm{MOCK})$-treated group whereas treatment with Ad5PTD(ASH1-SCG3-E1A) induced necrosis/apoptosis in the tumors. Fibrosis was not observed in either treatment group. Viral DNA copy number was higher in the tumors for the Ad5PTD(ASH1-SCG3-E1A)treated group whereas similar levels were found in the liver for both treatment groups. This indicates that viral replication was well restricted within the tumors. We detected hexon protein in the nuclei of tumor cells in the Ad5PTD(ASH1-SCG3-E1A)-treated group, which is in accordance with packaging of progeny virus (Wodrich et al., 2003). This further emphasizes in vivo viral replication. Taken together, the data are in accordance with the delayed tumor growth observed due to the oncolytic effect of Ad5PTD(ASH1-SCG3-E1A).

Chemotherapy is the standard treatment for neuroblastoma. However, treatment-induced drug resistance is a major problem with this approach and eventually the cancer-initiating cells are thought to become refractory to chemotherapeutic drugs. In our study, we established chemotherapeutic drug-insensitive neuroblastoma cell lines. The oncolytic adenoviruses were able to kill cisplatin-insensitive and cisplatin-sensitive SK-N-FI cells with equal efficiency. Doxorubicin-insensitive and etoposide-insensitive SH-SY-5Y cells were also killed by the oncolytic adenovirus even though the efficiency was lower compared with the corresponding drug-sensitive cell lines. Vincristine-insensitive $\mathrm{SH}$ SY-5Y cells were difficult to kill. Both beneficial (Yasui et al., 2011; Rein et al., 2012) and unfavorable effects have been reported (Hoti et al., 2006; Kanai et al., 2010) when oncolytic viruses are combined with chemotherapy drugs. Our experimental data suggest that patients treated with cisplatin would benefit from subsequent oncolytic adenovirus treatment, whereas patients treated with etoposide or doxorubicin may have a moderate beneficial effect and patients treated with vincristine may have less beneficial effect from oncolytic adenovirus treatment.

In conclusion, genetically engineered oncolytic adenoviruses are attractive anticancer therapeutics because of their ability to selectively replicate in tumor cells. Our data suggest that the oncolytic Ad5PTD(ASH1-SCG3-E1A) virus could be used to treat neuroblastoma, possibly together with mesenchymal stem cells, in conjunction with standard therapy.

\section{Acknowledgments}

Conceived and designed the experiments: C.J., D.Y., J.L., M.E. Performed the experiments and analyzed the data: C.J.,
D.Y., M.C., B.N., J.L. Wrote the paper: C.J., D.Y., J.L., M.E. All authors read and approved the final version of the manuscript. The authors thank Dr. Alkwin Wanders for grading the tumor sections for necrosis, Dr. Fredrik Hedborg for valuable discussions about neuroblastoma and neuroblastoma cell lines, Miss Lu Zhang for technical assistance, and Mohanraj Ramachandran for critical reading of the manuscript. The Swedish Cancer Society (10-0105 and 10-0552), the Swedish Children Cancer Foundation (PROJ10/027, NBCNSPDHEL10/013, PROJ11/062), Gunnar Nilsson's Cancer Foundation, the Swedish Research Council (K2013-55X22191-01-3), and the Marcus and Marianne Wallenberg's Foundation supported this work. The funders had no role in study design, data collection and analysis, decision to publish, or preparation of the manuscript.

\section{Author Disclosure Statement}

No competing financial interests exist.

\section{References}

Chen, H., Biel, M.A., Borges, M.W., et al. (1997). Tissue-specific expression of human achaete-scute homologue-1 in neuroendocrine tumors: Transcriptional regulation by dual inhibitory regions. Cell Growth Differ. 8, 677-686.

Cheng, W.S., Kraaij, R., Nilsson, B., et al. (2004). A novel TARPpromoter-based adenovirus against hormone-dependent and hormone-refractory prostate cancer. Mol. Ther. 10, 355-364.

Danielsson, A., Dzojic, H., Nilsson, B., and Essand, M. (2008). Increased therapeutic efficacy of the prostate-specific oncolytic adenovirus Ad[I/PPT-E1A] by reduction of the insulator size and introduction of the full-length E3 region. Cancer Gene Ther. 15, 203-213.

Essand, M. (2013). Virotherapy of neuroendocrine tumors. Neuroendocrinology 97, 26-34.

Garcia-Castro, J., Alemany, R., Cascallo, M., et al. (2010). Treatment of metastatic neuroblastoma with systemic oncolytic virotherapy delivered by autologous mesenchymal stem cells: An exploratory study. Cancer Gene Ther. 17, 476-483.

Gestblom, C., Grynfeld, A., Ora, I., et al. (1999). The basic helixloop-helix transcription factor dHAND, a marker gene for the developing human sympathetic nervous system, is expressed in both high- and low-stage neuroblastomas. Lab. Invest. 79, 67-79.

He, T.C., Zhou, S., Da Costa, L.T., et al. (1998). A simplified system for generating recombinant adenoviruses. Proc. Natl. Acad. Sci. U.S.A. 95, 2509-2514.

Hoehner, J.C., Gestblom, C., Hedborg, F., et al. (1996). A developmental model of neuroblastoma: Differentiating stromapoor tumors' progress along an extra-adrenal chromaffin lineage. Lab. Invest. 75, 659-675.

Hoti, N., Chowdhury, W., Hsieh, J.T., et al. (2006). Valproic acid, a histone deacetylase inhibitor, is an antagonist for oncolytic adenoviral gene therapy. Mol. Ther. 14, 768-78.

Iwasaki, I., Sugiyama, H., Kanazawa, S., and Hemmi, H. (2002). Establishment of cisplatin-resistant variants of human neuroblastoma cell lines, TGW and GOTO, and their drug cross-resistance profiles. Cancer Chemother. Pharmacol. 49, 438-444.

Kanai, R., Wakimoto, H., Cheema, T., and Rabkin, S.D. (2010). Oncolytic herpes simplex virus vectors and chemotherapy: Are combinatorial strategies more effective for cancer? Future Oncol. 6, 619-634. 
Kushner, B.H., Laquaglia, M.P., Bonilla, M.A., et al. (1994). Highly effective induction therapy for stage 4 neuroblastoma in children over 1 year of age. J. Clin. Oncol. 12, 2607-2613.

Leja, J., Dzojic, H., Gustafson, E., et al. (2007). A novel chromogranin-A promoter-driven oncolytic adenovirus for midgut carcinoid therapy. Clin. Cancer Res. 13, 2455-2462.

Leja, J., Nilsson, B., Yu, D., et al. (2010). Double-detargeted oncolytic adenovirus shows replication arrest in liver cells and retains neuroendocrine cell killing ability. PLoS One 5, e8916.

Li, L., Hung, A.C., and Porter, A.G. (2008). Secretogranin II: A key AP-1-regulated protein that mediates neuronal differentiation and protection from nitric oxide-induced apoptosis of neuroblastoma cells. Cell Death Differ. 15, 879-888.

London, W.B., Castleberry, R.P., Matthay, K.K., et al. (2005). Evidence for an age cutoff greater than 365 days for neuroblastoma risk group stratification in the Children's Oncology Group. J. Clin. Oncol. 23, 6459-6465.

Maris, J.M. (2010). Recent advances in neuroblastoma. N. Engl. J. Med. 362, 2202-2211.

Modak, S., and Cheung, N.K. (2010). Neuroblastoma: Therapeutic strategies for a clinical enigma. Cancer Treat. Rev. 36, 307-317.

Monclair, T., Brodeur, G.M., Ambros, P.F., et al. (2009). The International Neuroblastoma Risk Group (INRG) staging system: An INRG Task Force report. J. Clin. Oncol. 27, 298-303.

Ozawa, H., and Takata, K. (1995). The granin family-its role in sorting and secretory granule formation. Cell Struct. Funct. 20, 415-420.

Pesonen, S., Helin, H., Nokisalm, P., et al. (2010). Oncolytic adenovirus treatment of a patient with refractory neuroblastoma. Acta Oncol. 49, 117-119.

Portela-Gomes, G.M., Grimelius, L., and Stridsberg, M. (2010). Secretogranin III in human neuroendocrine tumours: A comparative immunohistochemical study with chromogranins A and B and secretogranin II. Regul. Pept. 165, 30-35.

Rein, D.T., Volkmer, A., Bauerschmitz, G., et al. (2012). Combination of a MDR1-targeted replicative adenovirus and chemotherapy for the therapy of pretreated ovarian cancer. J. Cancer Res. Clin. Oncol. 138, 603-610.

Rong, Y.P., Liu, F., Zeng, L.C., et al. (2002). Cloning and characterization of a novel human secretory protein: Secretogranin
III. Sheng Wu Hua Xue Yu Sheng $\mathrm{Wu} W u$ Li Xue Bao (Shanghai) 34, 411-417.

Stanton, R.J., McSharry, B.P., Armstrong, M., et al. (2008). Reengineering adenovirus vector systems to enable highthroughput analyses of gene function. Biotechniques 45, 659-662, 664-668.

Watt, F., Watanabe, R., Yang, W., et al. (2007). A novel MASH1 enhancer with N-myc and CREB-binding sites is active in neuroblastoma. Cancer Gene Ther. 14, 287-296.

Wodrich, H., Guan, T., Cingolani, G., et al. (2003). Switch from capsid protein import to adenovirus assembly by cleavage of nuclear transport signals. EMBO J. 22, 6245-6255.

Yan, X.D., Li, M., Yuan, Y., et al. (2007). Biological comparison of ovarian cancer resistant cell lines to cisplatin and Taxol by two different administrations. Oncol. Rep. 17, 1163-1169.

Yasui, T., Ohuchida, K., Zhao, M., et al. (2011). Adenoviral therapy is more effective in gemcitabine-resistant pancreatic cancer than in gemcitabine-sensitive cells. Anticancer Res. 31, 1279-1287.

Yu, D., Jin, C., Leja, J., et al. (2011). Adenovirus with hexon Tatprotein transduction domain modification exhibits increased therapeutic effect in experimental neuroblastoma and neuroendocrine tumors. J. Virol. 85, 13114-13123.

Yu, D., Jin, C., Ramachandran, M., et al. (2013). Adenovirus serotype 5 vectors with Tat-PTD modified hexon and serotype 35 fiber show greatly enhanced transduction capacity of primary cell cultures. PLoS One 8, e54952.

Address correspondence to: Dr. Magnus Essand Department of Immunology, Genetics, and Pathology Uppsala University SE-75185 Uppsala Sweden

E-mail: magnus.essand@igp.uu.se

Received for publication June 28, 2012; accepted after revision June 28, 2013.

Published online: July 26, 2013. 